\title{
Análise da estrutura dimensional da escala de automonitoria
}

\author{
Marcia Cristina Monteiro ${ }^{1}$ \\ Adriana Benevides Soares ${ }^{2}$ \\ ${ }^{1}$ União das Instituições Educacionais de São Paulo, SP, Brasil \\ https://orcid.org/0000-0003-3455-2690 \\ ${ }^{2}$ Universidade Salgado de Oliveira e Universidade do Estado do Rio de Janeiro, RJ, Brasil \\ https://orcid.org/0000-0003-2892-1808
}

\begin{abstract}
Resumo
As relações na universidade provavelmente são responsáveis por redes que facilitam os processos de aprendizagem e a permanência no Ensino Superior. O conceito de automonitoria pressupõe que as pessoas regulam a autoapresentação, adaptando ações de acordo com as pistas situacionais, o que muito facilitaria o processo de integração. Este estudo teve o objetivo de analisar a estrutura dimensional da Escala de Automonitoria por meio da análise de network. Participaram 500 alunos de instituições públicas e privadas de Ensino Superior, de ambos os sexos e idade entre 18 a 38 anos. Os dados foram coletados em sala de aula e os participantes assinaram o TCLE. A análise preservou os 18 itens e manteve dois fatores se aproximando do modelo em inglês. A escala apresenta evidências de validade que permite a utilização no contexto brasileiro. Estudos prospectivos podem viabilizar a análise das associações entre automonitoria e outras variáveis da adaptação acadêmica.
\end{abstract}

Palavras-chave: adaptação acadêmica, automonitoria, análise fatorial.

\section{Analysis of the dimensional structure of the self-monitoring scale}

\begin{abstract}
Relationships at the university are probably responsible for networks that facilitate learning processes and permanence in higher education. The concept of self-monitoring assumes that people regulate self-presentation, adapting actions according to situational cues, which would greatly facilitate the process of integration. This study had the objective of analyzing the dimensional structure of the Self-Monitoring Scale through network analysis. 500 students from public and private higher education institutions of both sexes and aged between 18 and 38 years participated. The data were collected in the classroom and the participants signed the CF. The analysis preserved the 18 items and maintained two factors approaching the English model. The scale presents evidence of validity that allows the use in the Brazilian context. Prospective studies may facilitate the analysis of the associations between self-monitoring and other variables of academic adaptation.
\end{abstract}

Keywords: academic adaptation, self-monitoring, factor analysis.

\section{Análisis de la estructura dimensional de la escala de auto-monitoreo}

\section{Resumen}

Las relaciones en las universidades son probablemente responsables de las redes que faciliten el aprendizaje de los procesos y la permanencia en la educación superior. El concepto de automonitoramento asume que las personas regulan la auto-presentación, adap- 
tando las acciones según la situación, que haría que el proceso de integración. Este estudio tuvo el objetivo de distinguir la dimensión estructural de la Escala de Auto-monitoreo a través de la red de análisis. De los estudiantes de edad y de edad entre 18 y 38 años y los participantes firmaron el FCI. El análisis conserva los 18 elementos y se mantiene dos factores acercándose al modelo inglés. La escala presenta evidencia de validación que permite utilizar en el contexto brasileño. Los estudios prospectivos pueden llevar el análisis de las asociaciones entre a auto-monitoreo y otras variables de la adaptación académica.

Palabras clave: adaptación académica, auto-monitoreo, análisis factorial.

A graduação é um momento importante na vida do estudante, já que envolve a busca da concretização de sonhos e expectativas almejadas com a nova etapa de vida e futura entrada, como profissional, no mercado de trabalho (Gomes \& Soares, 2013). Abrange ainda a escolha do curso e da instituição. Todo esse processo nem sempre é facilmente concretizado, haja vista que muitos estudantes vivenciam de forma desadaptativa as mudanças e desafios encontrados no Ensino Superior, o que pode gerar retenção ou mesmo a não permanência e conclusão da graduação (Soares, Pinheiro, \& Canavarro, 2015).

Para muitos estudantes, os obstáculos estarão presentes nos novos conteúdos dos diferentes componentes curriculares, no deslocamento, às vezes para localidades distantes da moradia, no cumprimento das exigências pedagógicas inerentes à graduação e nas novas interações com pares, docentes e gestores. As relações que os alunos desenvolvem provavelmente são responsáveis por redes que facilitam os processos de aprendizagem (Brandão, Bolsoni-Silva, \& Loureiro, 2017). Os pares, supostamente, diante das dificuldades, contribuem na superação dos entraves trazidos pelos conteúdos curriculares que demandam pesquisa e dedicação. Nesse sentido, se entende que muito facilitaria ao estudante saber controlar a sua forma de expressão de comportamento com colegas, professores e demais atores da comunidade universitária, ressaltando a importância da automonitoria.

O construto é definido como um traço de personalidade relacionado às diferenças individuais quanto à forma como a pessoa observa, regula e controla a autoimagem e a expressão do comportamento (Snyder, 1987). Com foco nessa sequência, Snyder (1974); Snyder e Gangestad (1986) desenvolvem a Escala de Automonitoria (versão de 25 itens e 18 itens, respectivamente) com o objetivo de que o instrumento mensure cinco componentes hipotéticos do construto de automonitoria: Adequação social de autoapresentação, Atenção à informação de comparação social, Controle da autoapresentação e comportamentos expressivos, Apresentações estratégicas de autoapresentação e estados afetivos e especificidade situacional de autoapresentação; Comportamento expressivo. Gangestad e Snyder (1985a) relatam correlações positivas significativas de três fatores rotacionados com a variável de classe latente: autocontrole da expressão do comportamento, domínio da presença de palco e autoapresentação direcionada ao outro. $\mathrm{O}$ primeiro refere-se à capacidade de se expressar publicamente, mas camuflando os reais motivos do comportamento. $\mathrm{O}$ segundo diz respeito a sentir-se à vontade em situações públicas, sendo o centro das atenções nos relatos e no entretenimento. Finalmente, o último fator refere-se à autorregulação do comportamento no intuito de expressar aquilo que o grupo espera da pessoa.

Com a expansão do conceito (Snyder, 1987), os autores da escala (Snyder \& Gangestad, 1986) incluíram também a forma como o indivíduo pensa sobre si mesmo, isto é, foram inseridos o autoconceito e as relações interpessoais. Neste sentido, as pessoas com altos escores em automonitoria se definem como pragmáticas e direcionadas pelas contingências situacionais e as exigências dos papéis sociais. Por outro lado, pessoas com baixos escores em automonitoria percebem-se como reguladas por fatores internos e atributos duradouros (Leone, 2006).

Entretanto, o cenário das pesquisas envolvendo o conceito de automonitoria e a mensuração do construto não é consensual. Briggs e Cheek (1988) exploraram as propriedades psicométricas dos escores nas versões original (25 itens) e revisada (18 itens) da Escala de 
Automonitoria. A pesquisa contou com 3 mil participantes e os autores identificaram dois fatores subjacentes aos escores nas Escalas de Automonitoria. Um dos fatores como sendo uma combinação de extroversão, confiança social e orientação instrumental e o outro como tendo direcionamento externo (direcionamento para o outro). Briggs e Cheek (1988) concluíram que nenhuma das versões (18 itens e 25 itens) da escala representam adequadamente o construto conforme definido por Snyder (1974, 1987). Essa conclusão baseou-se no conteúdo dos itens de cada fator, nos padrões de correlações entre os fatores e nas medidas de outros atributos, como por exemplo, a sociabilidade e a ambição. Além disso, os autores observaram que muitos dos itens sobre o "eu" na escala foram excluídos da versão revisada (18 itens). Consequentemente, a versão de 18 itens representaria apenas as diferenças individuais nas orientações interpessoais e não diferenças individuais sobre si, como sugerido por Snyder (1987). Em síntese, Briggs e Cheek (1988) sugerem que os fatores deveriam ser denominados de aquisitivo e autoprotetivo, em consonância com as estratégias de autoapresentação denominada de protetiva (adequação ao contexto) e aquisitiva (habilidade de modificar a autoapresentação).

Já Lennox e Wolfe (1984) argumentam que os resultados das análises fatoriais apresentam duas situações problemáticas. A primeira diz respeito ao fato que os fatores que emergem não correspondem conceitualmente as quatro das cinco dimensões hipotéticas que orientaram o desenvolvimento da Escala de Automonitoria original. Para os autores, apenas o fator direções externas (direcionamento para o outro) corresponde a um dos componentes conceituais (atenção à informação de comparação social). A segunda mostra que fatores que emergem de estudos analíticos envolvem padrões diferentes de correlações com outras variáveis. Escores de extroversão parecem estar relacionados ao comportamento gregário e a presença de confiança social, enquanto direções externas parecem se relacionar com timidez e falta de confiança social.
Lennox e Wolfe (1984) destacam ainda que as análises fatoriais e os diferentes critérios de interpretação indicam distintas soluções fatoriais (dois fatores, três fatores e quatro fatores). Dessa forma e com o objetivo de compreender a argumentação dos autores da escala (Snyder, 1974; Snyder \& Gangestad, 1986) sobre os cinco componentes hipotéticos propostos que guiaram o desenvolvimento da escala original e nas evidências de validade da escala, Lennox e Wolfe (1984) elaboram a Escala Revisada de Automonitoria utilizando itens consistentes com as dimensões conceituais latentes do construto de automonitoria (Snyder, 1974).

A Escala Revisada de Automonitoria destaca a diferença individual da sensibilidade na expressão do comportamento do outro, na habilidade de modificar a autoapresentação e na adequação social. A escala não apresenta escores relacionados à ansiedade e à extroversão/sociabilidade. Lennox e Wolfe (1984) chegaram a dois fatores: aquisição da autoapresentação, que reflete o desejo de ter aprovação social e a forma protetiva da autoapresentação, que reflete o desejo de evitar a desaprovação social. Os autores recomendam que as pesquisas sobre o tema retornem ao construto original de automonitoria, isto é, as diferenças individuais na autoapresentação e sugerem que a construção de evidências de validade seja um processo contínuo que envolva discussões calorosas da explicação e mensuração do construto, o que pode resultar em novas ideias e avanços na compreensão.

Por outro lado, Snyder e Gangestad (1986) argumentam sobre a evidência de predição nos escores totais da escala e assumem que a escala reflete um fator geral. Os pesquisadores apontam que técnicas fatoriais analíticas podem ser utilizadas para produzir um fator não rotacionado, no qual a maioria dos itens da escala carrega. Usando análises taxométricas, Gangestad e Snyder (1985b) fornecem evidências de que há uma discreta variável latente subjacente à variabilidade nos escores da Escala de Automonitoria e que esta variável representa mais variação nas respostas cognitivas, afetivas e comportamentais do que as pontuações na 
escala completa ou nos três fatores separados dentro da escala. Por meio de pesquisa com gêmeos, Gangestad e Snyder (1985a) encontram evidências de que o componente hereditário subjacente atua na variabilidade dos escores da Escala de Automonitoria e os escores dos fatores na escala compartilham uma quantidade substancial de influência genética.

Para Leone (2006), embora este intercâmbio entre grupos de estudiosos sobre automonitoria represente um debate efervescente, essa e outras trocas não são ainda as últimas palavras sobre as questões psicométricas. Na verdade, várias ferramentas analíticas foram sugeridas como meio de abordar a utilidade teórica das pontuações na Escala de Automonitoria (Leone, 2006). Essas ferramentas incluem, mas não se limitam, à análise fatorial confirmatória, equações estruturais e análise de classe latente. Se essas ou outras ferramentas analíticas são valiosas para explicar o significado das pontuações na Escala de Automonitoria, ainda não foram totalmente determinadas. $\mathrm{O}$ autor acrescenta que todas essas divergências são desafiadoras quando se pensa na metodologia aplicada (dicotomização de escores da escala e subescala versão de 25 itens e de 18, utilização de formato falso-verdadeiro e outros de resposta ao item do tipo Likert). Leone (2006) acrescenta que as variações processuais e de avaliação são tentativas para determinar a utilidade das diferenças métricas (total de escores versus escores das subescalas) na predição dos correlatos cognitivos, afetivos e comportamentais.

Mais recentemente, Wilmot (2015) realizou um estudo utilizando a escala na versão de 25 itens, a amostra original de Snyder (1974) (N=1.918) e uma amostra replicante com 2.951 participantes para retestar a estrutura latente de automonitoria por meio de procedimentos taxonométricos contemporâneos. As análises preliminares indicaram que o conjunto de indicadores de oito itens utilizados no estudo original não possui validade suficiente para detectar a estrutura categórica latente. Além disso, a subescala Direções Externas, um dos três indicadores derivados da análise analítica presentes na pesquisa original, também foi considerado inapropriado, devido a uma combinação de baixa validade e ortogonalidade em relação às suas subescalas. As duas escalas remanescentes, Ação e Extroversão, apresentam excelentes propriedades como indicadores, sendo submetidas a múltiplos procedimentos taxométricos e testes de consistência. Além disso, os resultados não sustentam os dados obtidos nas análises taxonométricas originais. Contrariamente, as curvas de comparação múltipla e a média da curva de comparação do índice de ajuste $(\mathrm{CCFI}=0,214)$ fornecem evidências fortes e convergentes de que a estrutura latente da automonitoria é dimensional e não categórica. $\mathrm{O}$ autor acrescenta que as dimensões encontradas indicam que o modelo convencional de automonitoria merece ser reexaminado e que os modelos teóricos, as práticas de medição e os procedimentos analíticos de dados que assumem a taxometria devem ser substituídos por conceituações dimensionais e procedimentos estatísticos correspondentes.

Em termos de pesquisa sobre automonitoria e adaptação à universidade um único estudo foi encontrado na literatura internacional até o momento. Guarino, Michael e Hocevar (1998) investigaram a integração de estudantes universitários. Contando com uma amostra de 380 alunos de ambos os sexos de uma faculdade norte-americana, os autores pesquisaram a integração tanto acadêmica como social dos estudantes. Utilizando a Escala de Automonitoria (Snyder, 1974), concluíram que os alunos com baixos escores em automonitoria apresentavam uma maior integração acadêmica, mostram-se mais envolvidos com as diferentes tarefas dos componentes curriculares, assim como estão atentos a oferta de atividades curriculares e extracurriculares que agreguem conhecimentos ao curso ou à futura carreira. $\mathrm{Na}$ amostra do estudo, os homens apresentaram escores mais elevados para integração social, mostrando mais interesse nas relações interpessoais e menos foco nos estudos.

A automonitoria autorregula o comportamento, o que supostamente contribui com a inserção do estudante aos novos grupos no Ensino Superior. Parte-se do princípio 
que os estudantes com altos escores em automonitoria são atentos às pistas do contexto e procuram adequar o comportamento conforme os interesses do grupo e aqueles com baixos escores são mais sensíveis as próprias concepções. Entretanto, o estudo de Guarino et al. (1998) sinaliza para o fato de que o aluno ou o próprio contexto educacional não associa o sucesso nas relações interpessoais com os processos de aprendizagem. Soares e Del Prette (2015), ao contrário, ressaltam que as aprendizagens que ocorrem no Ensino Superior são cognitivas e relacionais, o que auxiliaria o estudante no contexto universitário, como no profissional, e para a própria vida.

No cenário nacional, Monteiro e Soares (2019; submetido) realizaram uma análise exploratória e confirmatória, respectivamente, da Escala de Automonitoria. O primeiro estudo (2017) teve como objetivo a adaptação e a validação da Escala de Automonitoria na versão de 18 itens. A amostra contou com 200 estudantes universitários de Instituições de Ensino Superior (IES) particulares (50\%) e 50\% de IES públicas. A análise fatorial exploratória (AFE) indicou a extração de dois fatores e a exclusão de oito itens. Apesar do índice de confiabilidade obtido na escala $(0,92)$, os resultados não podem ser generalizados para um público mais amplo do que aquele formado por estudantes universitários. Além disso, alguns itens apresentaram valores pouco expressivos de correlação. O segundo estudo (Monteiro \& Soares, 2019; submetido) teve o objetivo de investigar a estrutura da Escala de Automonitoria (EA) adaptada e validada para a realidade brasileira (Monteiro \& Soares, 2017), além de avaliar a relação com o Inventário de Resolução de Problemas (IRPS) na EA. Participaram 400 estudantes de instituições de educação superior públicas e privadas, de diferentes graduações e de ambos os sexos. A análise fatorial confirmatória (AFC) da Escala de Automonitoria (EA) encontrou índices de ajuste que suportam a aceitabilidade do modelo. Já a modelagem por equações estruturais (MEE) mostrou que a habilidade em resolução de problemas (HRP) do IRPS apresentou impacto significativo e negativo nos níveis de automonitoria.

Diante dos questionamentos apresentados sobre a Escala de Automonitoria e pelos dados obtidos tanto na AFE e AFC realizadas (Monteiro \& Soares, 2019; submetido), este estudo tem o objetivo de analisar a estrutura dimensional da Escala de Automonitoria por meio da análise de network.

\section{Método}

\section{Participantes}

Participaram do estudo 500 alunos de instituições públicas e privadas de Ensino Superior situadas no Estado do Rio de Janeiro, sendo 100 (20\%) de instituições públicas. A amostra contou com estudantes de ambos os sexos e 115 (23\%) homens. A idade dos participantes foi entre 18 a 38 anos $(\mathrm{M}=23,8 ; \mathrm{DP}=3,2)$. A Tabela 1 apresenta o detalhamento da amostra por graduação, período e nível socioeconômico (NSE).

\section{Tabela 1}

Descrição da amostra por curso, período e NSE

\begin{tabular}{|l|l|}
\hline \multicolumn{1}{|c|}{ Curso } & \multicolumn{1}{|c|}{ Número de partipantes } \\
\hline Odontologia, Direito, Farmácia, & \\
Turismo, Biomedicina e Física & $1(0,2 \%)$ \\
Ciências Contábeis, Biologia e & $2(0,4 \%)$ \\
Letras & $4(0,8 \%)$ \\
Fisioterapia & $5(1 \%)$ \\
Ciências da Computação & $11(2,2 \%)$ \\
Administração & $16(3,2 \%)$ \\
Outros cursos & $20(4 \%)$ \\
Educação Física, Geografia e & $26(5,2 \%)$ \\
Engenharia & $29(5,8 \%)$ \\
Matemática & $38(7,6 \%)$ \\
Serviço Social & $45(9 \%)$ \\
Pedagogia & $118(23,6 \%)$ \\
História & $146(29,2 \%)$ \\
Enfermagem & \\
Psicologia & \\
\hline
\end{tabular}




\begin{tabular}{|l|c|}
\hline \multicolumn{2}{|c|}{ Período } \\
\hline Primeiro & $167(33,4 \%)$ \\
Segundo & $46(9,2 \%)$ \\
Terceiro & $98(19,6 \%)$ \\
Quarto & $26(5,2 \%)$ \\
Quinto & $52(13,8 \%)$ \\
Sexto & $21(4,2 \%)$ \\
Sétimo & $43(8,6 \%)$ \\
Oitavo & $24(4,8 \%)$ \\
Nono & $13(2,6 \%)$ \\
Décimo & $5(1 \%)$ \\
Décimo primeiro & $2(0,4 \%)$ \\
décimo segundo, décimo & $1(0,2 \%)$ \\
terceiro e décimo quarto & \\
\hline \multicolumn{2}{|c|}{ NSE } \\
\hline A & $58(11,6 \%)$ \\
B1 & $66(13,2 \%)$ \\
B2 & $139(27,8 \%)$ \\
C1 & $129(25,8 \%)$ \\
\hline D/E & $82(16,4 \%)$ \\
\hline
\end{tabular}

\section{Instrumento}

A Escala de Automonitoria é constituída de 18 itens e todos são dispostos em sentenças afirmativas com opção de marcação verdadeira ou falsa. A correção da escala estabelece que os itens 1, 2, 3, 7, 9, 11, 13, 14, 15 e 16 devem ser marcados como verdadeiros para os respondentes com baixa automonitoria, tendo o item valor 0 para a resposta verdadeira e 1 para falsa. O mesmo se aplica para os respondentes com alta automonitoria, sendo os itens 4, 5, 6, 8, 10, 12, 17 e 18 pontuados como verdadeiro tendo peso 1 e a resposta falsa com peso 0 .

\section{Procedimentos de coleta de dados}

Inicialmente foi realizado um contato prévio com diferentes instituições, solicitando a aplicação do instrumento. Os dados foram coletados nos momentos livres dos estudantes e em sala de aula com a prévia autorização das coordenações das graduações e dos docentes.

\section{Procedimentos éticos}

O presente estudo foi submetido e aprovado pelo Comitê de Ética em Pesquisa da Universidade Salgado de Oliveira. Todos os participantes assinaram o Termo de Consentimento Livre e Esclarecido (TCLE), que informava os objetivos da pesquisa e as regras de preservação do anonimato. O TCLE também informava o contato do pesquisador, caso o voluntário necessitasse para quaisquer esclarecimentos, garantindo-lhe o direito de retirada do consentimento a qualquer momento da pesquisa.

\section{Procedimento de análises dos dados}

A abordagem utilizada para a análise exploratória foi um modelo desenvolvido dentro da perspectiva da análise de network denominada Exploratory Graph Analysis (EGA) (Golino \& Epskamp, 2017), que se fundamenta no lasso (least absolute shrinkage and selection operator) método de análise de regressão que regulariza e seleciona variáveis a fim de aumentar a precisão e a interpretação do modelo estatístico pesquisado - com parâmetro de regularização extended Bayesian information criterion (EBIC) e estima o número de dimensões usando o Walktrap, um algoritmo de caminhada aleatória utilizado para reconhecer comunidades em redes (Machado, Vissoci, \& Epskamp, 2015). Segundo Golino e Epskamp (2017), a EGA propõe superar as restrições dos métodos tradicionais na estimação do número de dimensões estabelecidas por instrumentos utilizados na Psicologia e na Educação. Os autores afirmam que as estimações são obtidas por meio das correlações de um aglomerado de variáveis que explicam, no conjunto, um construto ou na variabilidade de dados multivariados sobre um fator latente. Golino e Epskamp (2017) ressaltam, ainda, que tradicionalmente são utilizados os métodos de eigenvalues, hipótese de máximo a posteriori (MAP) e índices de ajuste como Critério de Informação Bayesiano (BIC) e o Critério Esperado de Informação Bayesiano (EBIC). Desta forma, EGA estima as dimensões e determina os itens relacionados a cada dimensão com o objetivo de maior precisão de mensuração dos fatores no conjunto dos dados psicológicos. 


\section{Resultados}

A EGA foi utilizada na Escala de Automonitoria (Figura 1) e apresentou duas dimensões. Os nodos representam os itens da escala e as comunidades e os fatores ou dimensões estão destacados em duas cores, sendo a vermelha o Fator 1 e a azul o Fator 2. Denominou-se de Reações Internalizantes (Fator 1 - F1) os comportamentos que lidam com eventos de forma predominantemente fechada - as ideias e opiniões da pessoa são mantidas e esta respeita as suas concepções; Reações Externalizantes (Fator 2 - F2) por se referirem a comportamentos que respondem as demandas relacionais do ambiente e expressos prevalentemente de forma aberta - ideias, concepções e condutas são exteriorizadas publicamente. Os dois fatores estimados pela EGA mostram um modelo parcimonioso, apresentando as associações entre itens e fatores, além de preservar os 18 itens da escala, conforme o modelo original em inglês.

Figura 1. Network de correlação parcial estimada pela Exploratory Graph Analysis mostrando dois fatores da Escala de Automonitoria

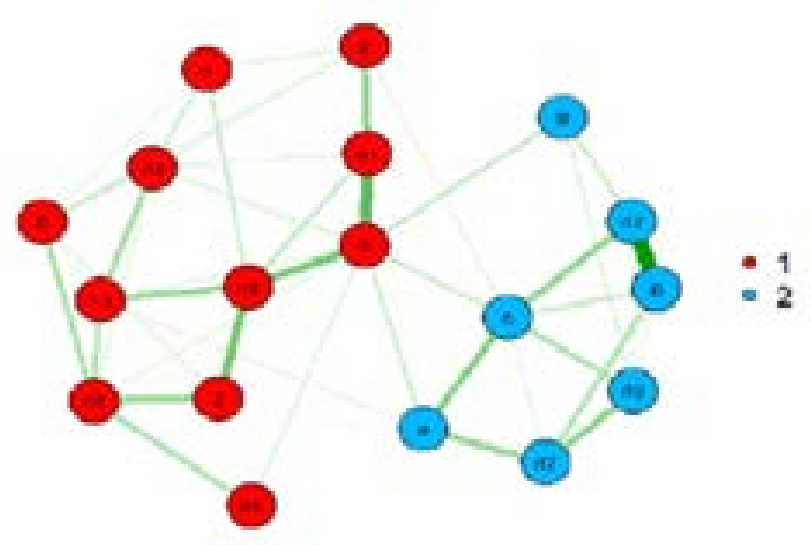

Realizou-se, com objetivo de comparação dos resultados, a mensuração por meio do modelo bifatorial Schmid-Leiman (Reise, 2012) obtendo-se também dois fatores específicos relacionados aos mesmos itens do obtido na EGA e mais um fator geral que explica os 18 itens da escala $(\mathrm{CFI}=0,97$; RMSEA $=0,03)$, conforme a Figura 2 .
Figura 2. Modelo Bifatorial da Escala de Automonitoria

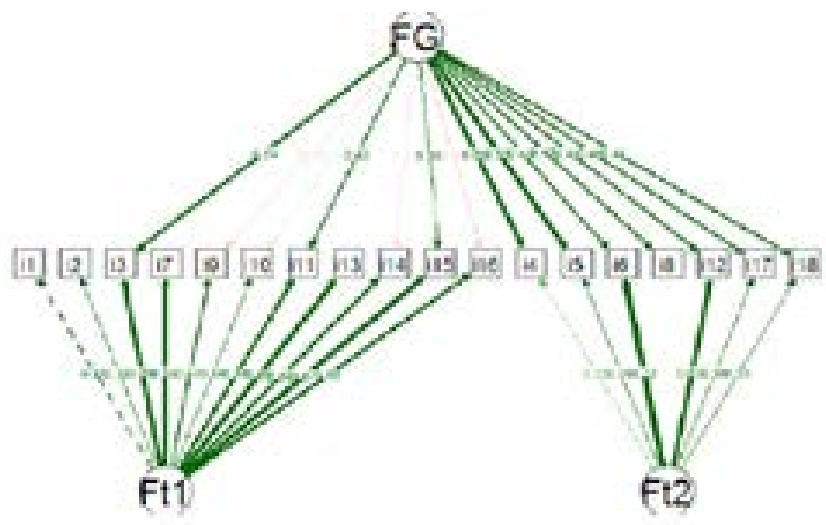

\section{Discussão}

O ingresso no Ensino Superior no Brasil vem se caracterizando por canditados que apresentam uma heterogeneidade de experiências pedagógicas e relacionais (Gomes \& Soares, 2013; Soares \& Del Prette, 2015; Soares, Pinheiro, \& Canavarro, 2015). Nessa diversidade, muitos estudantes experimentam, com dificuldades, as exigências inerentes ao Ensino Superior, como fazer novos relacionamentos com pares, lidar com a didática docente e defender ideias e opiniões. No cotidiano é comum observamos que alguns estudantes, mais do que outros, mudam o comportamento em função das pessoas e situações que os circundam. Muito facilitaria ao aluno no processo de adaptação ao novo contexto educacional o manejo adequado das relações interpessoais. Em outros termos, o discente que monitora a autoapresentação pode se relacionar de forma mais adequada com a comunidade universitária.

A automonitoria (Snyder, 1974; Snyder \& Gagestad, 1986) mostra-se com um aspecto facilitador na construção de novas amizades e sustentação dos vínculos já existentes. Os resultados obtidos na análise da Escala de Automonitoria mostraram que os respondentes preservam a ideia de semanterem em concordância com a maneira de conceberem e defenderem os princípios que regulam e norteam as suas intenções e ações. Procuram respeitar as motivações internas não considerando as movimentações externas que estejam 
presentes no momento. Outros procuram estar atentos às nuances do contexto e adequam as suas ideias e opiniões mediante as percepções obtidas. Para Snyder (1974), as pessoas com altos escores em automonitoria utilizam estratégias específicas para administrarem as autoapresentações a partir de pistas situacionais, respondendo de forma adaptada às contingências.

Nesse sentido, a escala construída e revisada (Snyder \& Gangestad, 1986) na versão de 18 itens propõe diferenciar indivíduos com alta e baixa automonitoria, sendo que os primeiros teriam mais capacidade e motivação de responderem às pistas situacionais. A análise realizada preservou os 18 itens da escala adaptada e validada para o português do Brasil. A EGA permitiu uma aproximação da escala em inglês a partir do momento que preservou os itens e manteve dois fatores, que originalmente foram denominados de Desempenho Público e Direcionamento para o Outro (Hoyle \& Lennox, 1991).

Os fatores encontrados podem indicar que os estudantes estão atentos às questões internas e externas que regem as relações interpessoais e há aqueles que procuram construir os relacionamentos atentos ou mesmo motivados pelas circunstâncias internas. Dessa forma, procuram se posicionar no contexto acadêmico norteando-se pelas concepções que acreditam ser os princípios relevantes para se colocarem perante o outro defendendo ideias e concepções, como exemplificado no item "Só defendo ideias em que já acredito". Outros apoiam-se nas circunstâncias externas, procurando estar atentos às pistas situacionais e adequando o seu posiciocionamento conforme às mudanças observadas no contexto, o que é corroborado pelas pesquisas na área (Guarino et al., 1998; Monteiro \& Soares, 2017) e exemplificado no item "Acho que faço um verdadeito espetáculo para impressionar ou entender os outros".

O presente estudo sustentou um modelo da Escala de Automonitoria sistematizado e em consonância com a literatura internacional sobre o modelo de dois fatores (Lennox \& Wolf, 1984; Hoyle \& Lennox, 1991) e esclarece aspectos que suscitaram questionamentos nos estudos de AFE e ACF (Monteiro \& Soares, 2017; submetido). Sublinhe-se que pesquisas envolvendo análises da escala enriquecem a discussão sobre a mesma e não se encerram em si (Leone, 2006), demonstrando que a escala possui rigor científico que possibilita indagações sistemáticas sobre as evidências de validade.

\section{Considerações finais}

O estudo analisou a estrutura dimensional da Escala de Automonitoria por meio da análise de network. A utilização da EGA manteve os 18 itens propostos para a escala e em conformidade com a versão original em inglês, além de indicar as associações entre os itens. A Escala de Automonitoria é pesquisada há mais de quatro décadas e ainda aponta um campo promissor em termos de investigação. Para a realidade nacional, por não ter sido encontrado outro instrumento que mensurasse o construto, o estudo de análise exploratória abre caminho para novas investigações sobre a escala, inclusive na inserção de itens vinculados à realidade brasileira.

Novas pesquisas sobre evidências de validade podem ser fundamentais, por se tratar de um processo contínuo. Outrossim, estudos com estudantes de outros estados da federação, considerando contextos culturais diversos, poderiam ser conduzidos e contribuir com o aperfeiçoamento da medida. Ademais, ressalta-se que a utilização da Escala de Automonitoria não se restringe à população universitária e pode ser aplicada em outros contextos, como no trabalho, no comportamento do eleitor e mesmo nas relações amorosas. Entretanto, os resultados obtidos permitem o reconhecimento da utilização da EA com estudantes universitários, o que possibilita estudos comparativos com versões em outros idiomas, além do original na língua inglesa. Pesquisas prospectivas podem viabillizar a análise das associações entre automonitoria e outras variáveis da adaptação acadêmica na população estudantil. 


\section{Referências}

Brandão, S. A., Bolsoni-Silva, A. T., \& Loureiro, S. R. (2017). Preditores da conclusão da graduação: Habilidades sociais, saúde mental, características acadêmicas. Paidéia, 27(66),117-125. http://dx.doi. org/10.1590/1982-43272766201714

Briggs, S. R., \& Cheek, J . M. (1988). On the nature of self-monitoring: Problems with assessment, problems with validity. Journal of Personality and Social Psychology, 54, 663-678. http://dx.doi.org/10.1037/0022$\underline{3514.54 .4 .663}$

Gangestad, S. \& Snyder, M. (1985a). "To carve nature at its joints": On the existence of discrete classes in personality. Psychological Review, 92, 317-349. https:// doi.org/10.1037/0033-295X.92.3.317

Gangestad, S. \& Snyder, M. (1985b). On the nature of self-monitoring: An examination of latent causal structure. In P. Shaver (Ed.), Review of Personality and Social Psychology (Vol. 6, pp. 65-86). Beverly Hills: Sage.

Golino, H. F., \&Epskamp, S. (2017). Exploratory graph analysis: A new approach for estimating the number of dimensions in psychological research. PLoS ONE, 12(6), e0174035. https://doi.org/10.1371/journal.pone.0174035

Gomes, G. \& Soares, A. B. (2013). Inteligência, habilidades sociais e expectativas acadêmicas no desempenho de estudantes universitários. Psicologia: Reflexão e Crítica, 26(4), 780-789. http://dx.doi.org/10.1590/S0102$\underline{79722013000400019}$

Guarino, A, Michael, W. B., \& Hocevar, D. (1998). Self-Monitoring and Student Integration of Community College Students. The Journal of Social Psychology, 138(6), 754-757. http://dx.doi. org/10.1080/00224549809603260

Hoyle, R. H., \& Lennox, R. D. (1991). Latent structure of self-monitoring. Multivariate Behavioral Research, 26(3), 511-540. https://doi.org/10.1207/s15327906mbr2603_8

Lennox, R. D., \& Wolfe, R. N. (1984). Revision of the self-monitoring scale. Journal of Personality and Social Psychology, 46, 1349-1364. https://doi.org/10.1037/0022$\underline{3514.46 .6 .1349}$

Leone, C. (2006). Self-monitoring: Individual differences in orientations to the social world. Journal of Personality, 74, 633-657. https://doi.org/10.1111/j. 1467-6494.2006.00387.x

Monteiro, M. C. , \& Soares, A. B. (2017). Adaptação e validação da Escala de Automonitoria para o português do Brasil. Avaliação Psicológica, 16(2), 169-175. https:// dx.doi.org/10.15689/AP.2017.1602.07.

Monteiro, M.C., \& Soares, A. B. (2019). Análise Confirmatória e Estudo de Convergência da Escala de Automonitoria. Manuscrito submetido para publicação.

Machado, W. L., Vissoci, J., \& Epskamp, S. (2015). Análise de rede aplicada psicometria e à avaliação psicológica. In C. S. Hutz, D. R. Bandeira, \& C. M. Trentini. Psicometria. (pp. 125-146). Porto Alegre: Artmed.

Reise, S. P. (2012). Invited Paper: The Rediscovery of Bifactor Measurement Models. Multivariate Behavioral Research,47(5), 667-696. https://doi. org/10.1080/00273171.2012.715555

Snyder, M. (1974). Self-monitoring of expressive behavior. Journal of Personality and Social Psychology, 30, 526-537. https://doi.org/10.1037/h0037039

Snyder, M. (1987). Public Appearances/Private Realities: The psychology of self-monitoring. New York: Freeman and Company.

Snyder, M., \& Gangestad, S. (1986). On the nature of self-monitoring: Matters of assessment, maters of validity. Journal of Personality and Social Psychology, 51, 125-139. https://doi.org/10.1037/0022-3514.51.1.125

Soares, A. B. \& Del Prette, Z. A. P. (2015). Habilidades sociais e adaptação à universidade: Convergências e divergências dos construtos. Análise Psicológica, 2(33), 139-151. http://dx.doi.org/10.14417/ap.911

Soares, A. M., Pinheiro, M. R., \& Canavarro, J. M. P.(2015). Transição e adaptação ao ensino superior e a demanda pelo sucesso nas instituições portuguesas. Psychologica, 58(2), 97-116. https://doi. org/10.14195/1647-8606_58-2_6

Wilmot, M. P. (2015). A contemporary taxometric analysis of the latent structure of self-monitoring. Psychological Assessment, 27(2), 353-364. https://doi. org/10.1037/pas0000030 
PSICO (PORTO ALEGRE) | V. 50, N. 3 | e-28544

Endereço para correspondência: Marcia

Cristina Monteiro (Av. Marechal Deodoro, 263,

Niterói, Rio de Janeiro, 24030-060; telefone (21)

33464330/994947988; marcialauriapsi@outlook.

com).

Recebido em: 14/9/2017.

Aprovado em: 14/5/2019.

Publicado em: $\mathrm{xx} / \mathrm{x} / \mathrm{xxxx}$

\section{Marcia Cristina Monteiro}

E-mail: marcialauriapsi@,outlook.com

https://orcid.org/0000-0003-3455-2690

Titulação Acadêmica: Doutora em Psicologia Social Afiliação Institucional: Grupo Universidade Brasil Faculdade Duque de Caxias e Universidade Salgado de Oliveira (UNIVERSO)

\section{Adriana Benevides Soares}

E-mail: adribenevides@gmail.com

https://orcid.org/0000-0003-2892-1808

Titulação Acadêmica: Doutora em Psicologia Cognitiva Afiliação Institucional: Universidade Salgado de Oliveira (UNIVERSO) e Universidade do Estado do Rio de Janeiro (UERJ) 